LA-UR-07-7067

SLAC-PUB-12893

arXiv:0710.3796[hep-ph]

October 2007

\title{
Sweet Spot Supersymmetry and Composite Messengers
}

\author{
Masahiro Ibe ${ }^{1,2}$ and Ryuichiro Kitano ${ }^{3}$ \\ ${ }^{1}$ Stanford Linear Accelerator Center, Stanford University, Stanford, CA 94309 \\ ${ }^{2}$ Physics Department, Stanford University, Stanford, CA 94305 \\ ${ }^{3}$ Theoretical Division T-8, Los Alamos National Laboratory, Los Alamos, NM 87545
}

\begin{abstract}
Sweet spot supersymmetry is a phenomenologically and cosmologically perfect framework to realize a supersymmetric world at short distance. We discuss a class of dynamical models of supersymmetry breaking and its mediation whose low-energy effective description falls into this framework. Hadron fields in the dynamical models play a role of the messengers of the supersymmetry breaking. As is always true in the models of the sweet spot supersymmetry, the messenger scale is predicted to be $10^{5} \mathrm{GeV} \lesssim M_{\text {mess }} \lesssim 10^{10} \mathrm{GeV}$. Various values of the effective number of messenger fields $N_{\text {mess }}$ are possible depending on the choice of the gauge group.
\end{abstract}

Work supported in part by US Department of Energy contract DE-AC02-76SF00515 


\section{Introduction}

If $N=1$ supersymmetry is hidden in nature, it helps us to understand the hierarchy between the strength of gravity and weak interactions and also the variety of matter fields and gauge forces in the standard model. It is, however, not straightforward to correctly hide supersymmetry at low energy. Especially, there has been a trouble in making the Higgs sector suitable for electroweak symmetry breaking, i.e., the $\mu$-problem. Also, the smallness of flavor mixing and CP violation has been considered as an unnatural aspect of the hypothesis.

Recently, the present authors carefully considered those problems, including cosmological one, and found a simple and realistic framework of supersymmetry breaking and mediation [1]. A small explicit breaking of the Peccei-Quinn (PQ) symmetry triggers supersymmetry to break down, and it induces $\mu$-term through (a generalized version of) the Giudice-Masiero mechanism [2]. The explicit breaking term also makes messenger fields massive via classical supergravity effects [3]. Correct sizes of gaugino and sfermion masses are obtained through their loop diagrams (gauge mediation 4, 5, 6, 7). The dangerous proton-decay operators of the mass dimensions four and five are forbidden by the PQ symmetry. A mechanism of producing dark matter of the universe is built-in; non-thermally produced gravitinos through the decay of the Polonyi field naturally explains the correct abundance [8]. This framework solves many known problems in supersymmetric model building, and have no apparent phenomenological or cosmological shortcomings left.

The framework, the sweet spot supersymmetry, is written in the language of the low-energy effective field theory. It is possible to construct various explicit models within this framework as ultraviolet (UV) completions, and each of those falls into a parameter point (or region) in the sweet spot supersymmetry. The parametrization can be done by four quantities: the number of messenger $N_{\text {mess }}$, the $\mu$-parameter, a gaugino mass, and the messenger scale $M_{\text {mess }}$. Once we specify those parameters, we can calculate the spectrum of superparticles by a simple program described in Ref. [1]. Conversely, by measuring those quantities at low energy experiments, we can obtain information on UV models.

In a recent paper [9], an economical UV model in this framework was proposed, that addresses the origin of the small explicit breaking term of the PQ symmetry. (It is called $\mathrm{U}(1)_{H}$ symmetry in Ref. [9].) It is found that the term can be non-perturbatively generated in a QCD-like theory ( $\mathrm{SU}\left(N_{c}\right)$ gauge theory with $N_{c}$ flavors), and the quark fields in that supersymmetric QCD play a role of the messenger fields. A parameter region of the sweet spot supersymmetry is identified

for this UV completion: $N_{\text {mess }}=5$ and $10^{11} \mathrm{GeV} \lesssim M_{\text {mess }} \lesssim 10^{13} \mathrm{GeV}$. The lower bound on the messenger scale is obtained from a consistency of the analysis. 
In this paper, we argue that this class of models predicts $M_{\text {mess }} \lesssim 10^{10} \mathrm{GeV}$, rather than $M_{\text {mess }} \gtrsim 10^{11} \mathrm{GeV}$ where the analysis in Ref. [9] is meaningful. Nevertheless, we find that there is a consistent effective description in terms of hadron fields in that case. We can find a supersymmetry breaking vacuum where gaugino/sfermion masses are generated by loop diagrams of hadronic messenger fields instead of elementary quarks. Generalizations with $\operatorname{Sp}\left(N_{c}\right)$ and $\mathrm{SO}\left(N_{c}\right)$ gauge theories are also discussed.

\section{Model}

We briefly review the framework of the sweet spot supersymmetry, and present a dynamical model of supersymmetry breaking which falls into this framework.

\subsection{Sweet spot supersymmetry}

The Lagrangian of the sweet spot supersymmetry is written in terms of the fields in the minimal supersymmetric standard model (MSSM) (chiral superfields $\Phi_{\text {MSSM }}$ and gauge fields $W_{\alpha}$ ), the Goldstino field $S$, and the messenger fields $f$ and $\bar{f}$ that have quantum numbers of the standard model gauge group. . It is defined by the supergravity Lagrangian with a Kähler- and a superpotential, $K$ and $W$ :

$$
\begin{aligned}
K= & \Phi_{\mathrm{MSSM}}^{\dagger} \Phi_{\mathrm{MSSM}}+S^{\dagger} S+f^{\dagger} f+\bar{f}^{\dagger} \bar{f} \\
& -\frac{\left(S^{\dagger} S\right)^{2}}{\Lambda^{2}}+\left(\frac{c_{\mu} S^{\dagger} H_{u} H_{d}}{\Lambda}+\text { h.c. }\right)-\frac{c_{H} S^{\dagger} S\left(H_{u}^{\dagger} H_{u}+H_{d}^{\dagger} H_{d}\right)}{\Lambda^{2}}, \\
W= & W_{\text {Yukawa }}\left(\Phi_{\mathrm{MSSM}}\right)+m^{2} S+k S f \bar{f}+w_{0} .
\end{aligned}
$$

In the Kähler potential, there are direct interaction terms between the Goldstino fields $S$ and the Higgs fields $H_{u}$ and $H_{d}$ suppressed by a 'cut-off' scale $\Lambda$. $\left(c_{\mu}\right.$ and $c_{H}$ are $O(1)$ coefficients.) f] These interactions are responsible for generating the $\mu$-term and the soft mass terms for the Higgs fields. In the superpotential, a linear term of $S$ represents the source term of the $F$ component of $S$. The interaction term between $S$ and messenger fields $f$ and $\bar{f}$ is responsible for gauge mediation. The constant term $w_{0}$ is needed to cancel the cosmological constant such that $w_{0}=m_{3 / 2} M_{\mathrm{Pl}}^{2}=m^{2} M_{\mathrm{Pl}} / \sqrt{3}$, where $m_{3 / 2}$ is the gravitino mass. This is the most general Lagrangian with the PQ-symmetry, $P Q(S)=2, P Q\left(H_{u}\right)=P Q\left(H_{d}\right)=1$, and $P Q\left(m^{2}\right)=-2$,

\footnotetext{
${ }^{*}$ The Lagrangian of the sweet spot supersymmetry is presented in Ref. [1] as the one after integrating out the messenger fields $f$ and $\bar{f}$. The original form contains terms in Eqs. (6) and (7) instead of those involving $f$ and $\bar{f}$. They are, of course, equivalent.

${ }^{\dagger}$ We assumed that at least first two generations of the quark and lepton superfields are weakly coupled in a theory above the scale $\Lambda$ in order to avoid flavor changing or CP violating interactions suppressed by the scale $\Lambda$. For an explicit example of such microscopic models, see Ref. [1, 10].
} 
where $m^{2}$ represents the small explicit breaking parameter. Smallness of the supersymmetry breaking scale and also of the $\mu$-parameter are controlled by this parameter.

Obviously, there is a supersymmetric minimum in this model where

$$
\langle S\rangle=0,\langle f \bar{f}\rangle=-m^{2} / k .
$$

However, we can find a local minimum with broken supersymmetry if the value of $k$ is small enough [3]. From the above $K$ and $W$, we obtain a scalar potential for the $S$ field:

$$
V(S)=m^{4}\left(\frac{4}{\Lambda^{2}}|S|^{2}+\frac{k^{2} N}{(4 \pi)^{2}} \log \left(\frac{k^{2}|S|^{2}}{\Lambda^{2}}\right)\right)-\left(2 m_{3 / 2} m^{2} S+\text { h.c. }\right) .
$$

The logarithmic term is a loop correction from the interaction term, $k S f \bar{f}$, and $N$ is a number of fields running in the loop. For example, $N=5$ if the messenger fields $f$ and $\bar{f}$ transform as 5 and $\overline{5}$ representations under $\mathrm{SU}(5)$ symmetry $\left((3,1)_{-1 / 3} \oplus(1,2)_{1 / 2}\right.$ and $(\overline{3}, 1)_{1 / 3} \oplus(1,2)_{-1 / 2}$ under the standard model gauge group). The linear term, $2 m_{3 / 2} m^{2} S$, is a supergravity effect; this is a soft supersymmetry breaking term associated with the linear term in the superpotential in Eq. (2). Once we ignore the logarithmic term, the minimum is at

$$
\langle S\rangle=\frac{\sqrt{3} \Lambda^{2}}{6 M_{\mathrm{Pl}}} .
$$

This makes the messenger fields massive, and thus stabilizes the $f \bar{f}$ direction. Supersymmetry is broken by $F_{S} \simeq m^{2}+k\langle f \bar{f}\rangle=m^{2}$. For a large value of $k$, however, this local minimum disappears because the quantum correction becomes stronger than the supergravity effects. As we will see in Section 3, the condition that there is a meta-stable supersymmetry breaking vacuum provides an upper bound on the messenger scale, $M_{\text {mess }}=k\langle S\rangle$.

By integrating out those massive messenger fields, we obtain terms responsible for the gaugino and sfermion masses (gauge mediation) [11]:

$$
f_{\text {kin }} \ni-\frac{N_{\text {mess }}}{(4 \pi)^{2}} \log S W^{\alpha} W_{\alpha},
$$

for the gauge kinetic function, and

$$
K \ni-\frac{4 g^{4} N_{\text {mess }}}{(4 \pi)^{4}} C_{2}(R)\left(\log |S|^{2}\right) \Phi_{\mathrm{MSSM}}^{\dagger} \Phi_{\mathrm{MSSM}},
$$

with $N_{\text {mess }}$ the number of the messenger fields $\left(N_{\text {mess }}=1\right.$ for a pair of 5 and $\overline{5}$ representations of $\mathrm{SU}(5))$. With the non-vanishing value of $F_{S}$ and $\langle S\rangle$, we obtain gaugino/sfermion masses through the above interaction terms.

There are two dimensionful parameters in this model: $\Lambda$ and $m_{3 / 2}\left(=m^{2} /\left(\sqrt{3} M_{\mathrm{Pl}}\right)=\right.$ $\left.w_{0} / M_{\mathrm{Pl}}^{2}\right)$. The interesting discovery in Ref. [1] is that there is a sweet spot in the two dimensional parameter space $\left(m_{3 / 2}, \Lambda\right)$ where everything works out fine. The choice is $\left(m_{3 / 2}, \Lambda\right) \sim$ 
$\left(1 \mathrm{GeV}, 10^{16} \mathrm{GeV}\right)$ with which we obtain correct sizes of the $\mu$-term, gaugino and sfermion masses, and the abundance of gravitino dark matter. The fact that $\Lambda$ is at the grand unification scale is also an interesting coincidence.

\section{$2.2 \quad$ A model of dynamical supersymmetry breaking}

A part of the above Lagrangian,

$$
\begin{array}{ll}
K & \ni \quad S^{\dagger} S-\frac{\left(S^{\dagger} S\right)^{2}}{\Lambda^{2}} \\
W & \ni m^{2} S
\end{array}
$$

provides an effective description of a quite general class of supersymmetry breaking models. As long as the Goldstino superfield (a field or a combination of fields which gets $F$-component VEV) is weakly coupled in the actual supersymmetry breaking model, the above Lagrangian is obtained by integrating out other massive fields in the model. $\mathrm{f}$ The $\Lambda$ parameter represents the strength of the self interactions of the Goldstino and $m^{2}$ is the size of the supersymmetry breaking.

We consider a simple example of such models where the superpotential terms in Eq. (2), $m^{2} S+k S f \bar{f}$, are replaced with a single term [9],

$$
W \ni k S(F \bar{F})
$$

where $F$ and $\bar{F}$ transforms as 5 and $\overline{5}$ representations of an $\mathrm{SU}(5)_{F}$ group (which contains the standard model gauge group as a subgroup). They also have quantum numbers (5 and $\overline{5})$ of another gauge group $\mathrm{SU}(5)_{H}$ which becomes strongly coupled at an energy scale $\Lambda_{\mathrm{dyn}}$. In the above term, the indices of $\mathrm{SU}(5)_{H}$ and $\mathrm{SU}(5)_{F}$ are contracted in $(F \bar{F})$.

We assume here that the gauge interaction of $\mathrm{SU}(5)_{H}$ becomes strong in the regime where the masses of messenger particles, $M_{\text {mess }}$, are not important, i.e., $M_{\text {mess }}<\Lambda_{\text {dyn }}$. In fact, in Ref. [9], it has been claimed that this regime is incompatible with the mechanism of supersymmetry breaking and mediation since the meta-stable vacuum in Eq. (可) disappears. We show, however, that we still have the gravitationally stabilized vacuum where supersymmetry is spontaneously broken. Furthermore, we will see in the next Section that $M_{\text {mess }}$ is almost always lower than the dynamical scale $\Lambda_{\text {dyn }}$ for the vacuum to be meta-stable.

\footnotetext{
${ }^{\ddagger}$ In general, if there is no (approximate) symmetry under which $S$ is charged in a supersymmetry breaking model, there can be a cubic term in the Kähler potential, $K \ni S^{\dagger} S^{2}+$ h.c.. However, such terms can be shifted away by an appropriate field redefinition $S \rightarrow S+c$. In the case of the sweet spot supersymmetry, the presence of the approximate PQ-symmetry is assumed. That restricts the form of Kähler and superpotential to be the ones in Eqs. (11) and (㫙).
} 
Below the scale $\Lambda_{\text {dyn }}$, there is an effective description of the theory in terms of meson $M_{i j} \sim$ $F_{i} \bar{F}_{j}$ and baryon fields $B \sim F^{5}$ and $\bar{B} \sim \bar{F}^{5}$. The indices $i, j(=1-5)$ are those of $\mathrm{SU}(5)_{F}$. The effective superpotential is given by

$$
W=k S \cdot \operatorname{Tr} M+X\left(\operatorname{det} M-B \bar{B}-\left(\Lambda_{\mathrm{dyn}}^{2} / 5\right)^{5}\right)
$$

where a Lagrange multiplier $X$ is introduced in order to ensure the quantum modified constraint to be satisfied [12]. We can find a meta-stable vacuum in the meson branch, $\operatorname{det} M=\left(\Lambda_{\text {dyn }}^{2} / 5\right)^{5}$. By solving the constraint around the point $M_{i j}=\Lambda_{\mathrm{dyn}}^{2} \delta_{i j} / 5$, we obtain

$$
\operatorname{Tr} M=\Lambda_{\text {dyn }}^{2}+\frac{1}{2} \frac{\operatorname{Tr} \delta M^{2}}{\Lambda_{\text {dyn }}^{2} / 5}+\frac{B \bar{B}}{\left(\Lambda_{\text {dyn }}^{2} / 5\right)^{4}}+\cdots,
$$

where $\delta M$ is the traceless part of the matrix $M$. We have neglected higher order terms in the field expansion. The effective superpotential below the scale $\Lambda_{\text {dyn }}$ is then given by

$$
W_{\text {eff }}=k \Lambda_{\text {dyn }}^{2} S+S\left(\frac{\hat{k}_{M}}{2} \operatorname{Tr} \delta \hat{M}^{2}+\hat{k}_{B} \hat{B} \hat{\bar{B}}\right) .
$$

The fields $\delta \hat{M}, \hat{B}$, and $\hat{\bar{B}}$ are canonically normalized fields. Through this normalization procedure, $O(1)$ uncertainties arise in the coupling constants $\hat{k}_{M} \sim \hat{k}_{B} \sim k$.

The effective superpotential above is exactly the one in Eq. (2) by the identifications of $m^{2} \sim k \Lambda_{\text {dyn }}^{2}, k \sim \hat{k}_{M}, f \sim \delta \hat{M}$ and $\bar{f} \sim \delta \hat{M}$. The baryon fields do not contribute to the gaugino/sfermion masses since they are singlet under the standard model gauge group. The field $\delta \hat{M}$, on the other hand, transforms as the adjoint representation under the $\operatorname{SU}(5)_{F}$ flavor group. (The quantum numbers under the standard model gauge group are $(8,1)_{0} \oplus(1,3)_{0} \oplus$ $(3,2)_{-5 / 6} \oplus(\overline{3}, 2)_{5 / 6} \oplus(1,1)_{0}$. $)$ By integrating out those meson fields, the terms in Eqs. (區) and (7) are obtained with $N_{\text {mess }}=5$. This is consistent with the anomaly matching condition for $\mathrm{U}(1)_{\mathrm{PQ}}-\mathrm{SU}(5)_{F}-\mathrm{SU}(5)_{F}$.

We need to make sure that the strong dynamics does not destabilize the vacuum in Eq. (5). Through the interaction term, $k S F \bar{F}$, it is expected to appear higher dimensional operators in the Kähler potential such as

$$
\delta K \sim \frac{N}{(4 \pi)^{2}} \frac{|k S|^{4}}{\Lambda_{\mathrm{dyn}}^{2}}
$$

where $N=25$. However, the effect of this term is smaller than that of the term $-\left(S^{\dagger} S\right)^{2} / \Lambda^{2}$ in Eq. (8) if

$$
k \lesssim 3 \times 10^{-3}\left(\frac{N}{25}\right)^{-1 / 5}\left(\frac{m_{3 / 2}}{1 \mathrm{GeV}}\right)^{1 / 5}\left(\frac{\Lambda}{1 \times 10^{16} \mathrm{GeV}}\right)^{-2 / 5}
$$


Here we have used a relation, $m^{2}=k \Lambda_{\text {dyn }}^{2}=\sqrt{3} m_{3 / 2} M_{\mathrm{Pl}}$. As we will see later, the above condition is always satisfied when $M_{\text {mess }}<\Lambda_{\text {dyn }}$ that we have already assumed.

There is a lower bound on the messenger scale by a condition that the messenger fields should not be tachyonic:

$$
M_{\mathrm{mess}}^{2}=\hat{k}_{M}^{2}\langle S\rangle^{2}>\hat{k}_{M} F_{S}
$$

Thus, we obtain

$$
M_{\text {mess }}=\hat{k}_{M}\langle S\rangle>\frac{F_{S}}{\langle S\rangle}=3 \times 10^{5} \mathrm{GeV}\left(\frac{m_{3 / 2}}{1 \mathrm{GeV}}\right)\left(\frac{\Lambda}{1 \times 10^{16} \mathrm{GeV}}\right)^{-2} .
$$

We will examine in the next Section whether we have a consistent parameter region.

\section{Upper bound on the messenger scale}

We derive an upper bound on the messenger scale from the stability of the vacuum in Eq. (5). From the discussion, we will learn that the messenger scale is almost always lower than the dynamical scale $\Lambda_{\text {dyn }}$, consistent with the assumption made in the previous section.

In order to derive an upper bound on the messenger scale we first consider a region with $M_{\text {mess }}>\Lambda_{\text {dyn }}$. In this case, the quark fields $F$ and $\bar{F}$ can be integrated out without considering the non-perturbative effects. The phenomenon of supersymmetry breaking can be understood in a slightly different way in this regime. Below the scale $M_{\text {mess }}$, the theory matches to the pure supersymmetric $\mathrm{SU}(5)_{H}$ gauge theory. Eventually at a scale $\Lambda_{\text {eff }}$, the superpotential acquires a contribution from the gaugino condensation, $W \ni \Lambda_{\text {eff }}^{3}$. Now, by a matching condition of the gauge coupling constant at the scale $M_{\text {mess }}$, we can see that this term has a dependence on the

field value of $S: \Lambda_{\text {eff }}^{3}=M_{\text {mess }} \Lambda_{\text {dyn }}^{2}=k S \Lambda_{\text {dyn }}^{2}$. This is the linear term of $S$ in Eq. (2) which causes supersymmetry breaking by $F_{S}=m^{2}=k \Lambda_{\text {dyn }}^{2}[9]$.

The quantum corrections to the scalar potential of $S$ can be calculated perturbatively in the picture where $F$ and $\bar{F}$ are elementary fields. It is simply the logarithmic term in Eq. (四) with $N=25$. A condition to have a local minimum in the scalar potential (蛋) is

$$
\frac{1}{3 M_{\mathrm{Pl}}^{2}}-\frac{4}{\Lambda^{2}} \frac{k^{2} N}{(4 \pi)^{2}}>0
$$

from which the bound on $k$ is obtained to be

$$
k<3 \times 10^{-3}\left(\frac{N}{25}\right)^{-1 / 2}\left(\frac{\Lambda}{1 \times 10^{16} \mathrm{GeV}}\right) .
$$


Therefore, with the VEV of $S$ in Eq. (5), we obtain the upper bound on the messenger scale to be

$$
M_{\text {mess }}<4 \times 10^{10} \mathrm{GeV}\left(\frac{N}{25}\right)^{-1 / 2}\left(\frac{\Lambda}{1 \times 10^{16} \mathrm{GeV}}\right)^{3}
$$

On the other hand, the dynamical scale $\Lambda_{\text {dyn }}$ has a relation to the $m^{2}$ parameter:

$$
m^{2}=k \Lambda_{\mathrm{dyn}}^{2}=\sqrt{3} m_{3 / 2} M_{\mathrm{Pl}}
$$

From this, we obtain

$$
\Lambda_{\text {dyn }}=4 \times 10^{10} \mathrm{GeV}\left(\frac{k}{3 \times 10^{-3}}\right)^{-1 / 2}\left(\frac{m_{3 / 2}}{1 \mathrm{GeV}}\right)^{1 / 2} .
$$

From Eqs. (18), (19) and (21), we conclude that the messenger scale is lower than the dynamical scale unless the bound in Eq. (18) is saturated. Note that we cannot go far from the sweet spot values of $m_{3 / 2}$ and $\Lambda$, otherwise the natural solution to the $\mu$-problem is spoiled.

This discussion justifies the assumption $M_{\text {mess }} \lesssim \Lambda_{\text {dyn }}$. In this regime, Eq. (18) should be understood as a condition for the coupling constants $\hat{k}_{M}$ and $\hat{k}_{B}$ rather than for the fundamental coupling constant $k$. Then, by a relation $\hat{k}_{M} \sim \hat{k}_{B} \sim k$, the inequality in Eq. (19) just results in a consistency condition: $M_{\text {mess }} \lesssim \Lambda_{\text {dyn }}$. The bound in Eq. (18) (barring $O(1)$ ambiguities in the relation between $k$ and $\hat{k}_{M}$ ) is identical to the previously obtained constraint in Eq. (14) which ensures the stability of the potential against corrections from the strong dynamics.

In summary, we have obtained a consistent region

$$
10^{5} \mathrm{GeV} \lesssim M_{\text {mess }} \lesssim 10^{10} \mathrm{GeV}
$$

for the messenger scale, where the hadron picture is appropriate for the analysis. Note, however, that this prediction is generally true in any models of the sweet spot supersymmetry. The only non-trivial prediction of this model is $N_{\text {mess }}=5$. In the next section, we examine the same class of models with different strong gauge groups. We find those models predict different values of $N_{\text {mess }}$.

\section{$4 \quad \operatorname{Sp}\left(\mathrm{N}_{\mathrm{c}}\right)$ and $\mathrm{SO}\left(\mathrm{N}_{\mathrm{c}}\right)$ models}

The mechanism of supersymmetry breaking and its mediation works also in $\operatorname{Sp}\left(N_{c}\right)$ and $\mathrm{SO}\left(N_{c}\right)$

gauge theories instead of $\mathrm{SU}(5)_{H}$. In order for the matching condition, $\Lambda_{\mathrm{eff}}^{3}=M_{\mathrm{mess}} \Lambda_{\mathrm{dyn}}^{2}$, to hold, gauge groups are determined to be $\mathrm{Sp}(4)$ or $\mathrm{SO}(12)$. 
The discussion is almost the same for the $\operatorname{Sp}(4)$ case. We introduce $F$ and $\bar{F}$, that are $(8,5)$ and $(8, \overline{5})$ under $\mathrm{Sp}(4)_{H} \times \mathrm{SU}(5)_{F}$ group. Again, the standard model gauge group is a subgroup of the $\mathrm{SU}(5)_{F}$ global symmetry. We assume an interaction term, $W \ni k S(F \bar{F})$, where both $\mathrm{Sp}(4)_{H}$ and $\mathrm{SU}(5)_{F}$ indices are appropriately contracted in $(F \bar{F})$. Below the dynamical scale of the $\mathrm{Sp}(4)_{H}$ gauge theory, $\Lambda_{\text {dyn }}$, the theory is described by meson fields, $M$. The superpotential is

$$
W=k S \cdot \operatorname{Tr} M_{F \bar{F}}+X\left(\operatorname{Pf} M-\left(\Lambda_{\mathrm{dyn}}^{2} / 5\right)^{5}\right)
$$

with $X$ a Lagrange multiplier [13. The meson field $M$ is a $10 \times 10$ antisymmetric matrix:

$$
M=\left(\begin{array}{cc}
M_{F F} & M_{F \bar{F}} \\
-M_{F \bar{F}}^{T} & M_{\bar{F} \bar{F}}
\end{array}\right) .
$$

The submatrix $M_{F F}, M_{\bar{F} \bar{F}}$, and $M_{F \bar{F}}$ transform as 10, $\overline{10}$, and $1+24$ under the $\mathrm{SU}(5)_{F}$ flavor group, respectively. By solving the constraint and canonically normalizing the fields, we obtain

$$
W_{\text {eff }}=k \Lambda_{\text {dyn }}^{2} S+S\left(\hat{k}_{F F} \operatorname{Tr}\left(\hat{M}_{F F} \hat{M}_{\bar{F} \bar{F}}\right)+\frac{\hat{k}_{F \bar{F}}}{2} \operatorname{Tr} \delta \hat{M}_{F \bar{F}}^{2}\right) .
$$

The effective number of messengers are $N_{\text {mess }}=8$ in this case.

The case with an $\mathrm{SO}(12)$ gauge group is essentially the same, yet a little bit more complicated. The quarks $F$ and $\bar{F}$ transform as $(12,5)$ and $(12, \overline{5})$ this time. Below the dynamical scale, the effective theory is a $\mathrm{U}(1)$ gauge theory with superpotential:

$$
W=k S \cdot \operatorname{Tr} M_{F \bar{F}}+\left(\operatorname{det} M-\left(\Lambda_{\mathrm{dyn}}^{2} / 5\right)^{10}\right) E^{+} E^{-},
$$

near a point $\operatorname{det} M=\left(\Lambda_{\mathrm{dyn}}^{2} / 5\right)^{10}$. The fields $E^{ \pm}$are dyons [14]. The meson field $M$ is a $10 \times 10$ symmetric matrix:

$$
M=\left(\begin{array}{cc}
M_{F F} & M_{F \bar{F}} \\
M_{F \bar{F}}^{T} & M_{\bar{F} \bar{F}}
\end{array}\right)
$$

The submatrix $M_{F F}, M_{\bar{F} \bar{F}}$, and $M_{F \bar{F}}$ transform as $15, \overline{15}$, and $1+24$ under the $\mathrm{SU}(5)_{F}$ flavor group, respectively. By turning on the $\mathrm{VEV}$ of $S$, the minimum of the potential is at

$$
\begin{aligned}
\left\langle M_{F \bar{F}}\right\rangle & =\left(\Lambda_{\mathrm{dyn}}^{2} / 5\right) \delta_{i j}, \\
\left\langle E^{+} E^{-}\right\rangle & =-\frac{k S}{2\left(\Lambda_{\mathrm{dyn}}^{2} / 5\right)^{9}} .
\end{aligned}
$$

In this vacuum, the $\mathrm{U}(1)$ symmetry is Higgsed at the scale $\left(k\langle S\rangle \Lambda_{\text {dyn }}\right)^{1 / 2}$ and the trace part of $M_{F \bar{F}}$ and $E^{ \pm}$obtain masses (or eaten by the $\mathrm{U}(1)$ gauge field) and decouple. The effects of 
those massive particles on the $S$ potential are always smaller than the term $K \ni-\left(S^{\dagger} S\right)^{2} / \Lambda^{2}$ for $M_{\text {mess }}<\Lambda_{\text {dyn }}$. Below the scale $\left(M_{\text {mess }} \Lambda_{\text {dyn }}\right)^{1 / 2}$, the effective superpotential is

$$
W_{\text {eff }}=k \Lambda_{\text {dyn }}^{2} S+S\left(\hat{k}_{F F} \operatorname{Tr}\left(\hat{M}_{F F} \hat{M}_{\bar{F} \bar{F}}\right)+\frac{\hat{k}_{F \bar{F}}}{2} \operatorname{Tr} \delta \hat{M}_{F \bar{F}}^{2}\right) .
$$

Here, we canonically normalized fields. Again, this is the superpotential of the sweet spot supersymmetry. As anticipated, the effective number of messengers is $N_{\text {mess }}=12$ in this $\mathrm{SO}(12)$ model.

In fact, there is another branch in the $\mathrm{SO}(12)$ model where the superpotential is given by

$$
W=k S \cdot \operatorname{Tr} M_{\mathrm{F} \overline{\mathrm{F}}}+M_{i j} q_{i}^{+} q_{j}^{-}
$$

where $q$ 's are monopoles. There is no supersymmetry breaking vacuum in this branch. This is consistent with the fact that gaugino condensation cancels and $W_{\text {eff }}=0$ in this case.

Although these models are similar to the IYIT model of supersymmetry breaking [15], there are essential differences. In the IYIT model, we need to introduce gauge singlet fields for each flat direction in order to kill all the supersymmetric vacuum. In the model presented in this paper, we introduced only one singlet field $S$. Therefore, there is a supersymmetric vacuum at $S=0$ since we do not fix all the flat directions. However, by the help of an external dynamics, i.e., supergravity interactions, $S$ can be stabilized away from the supersymmetric vacuum.

\section{Acknowledgments}

The work of MI was supported by the U.S. Department of Energy under contract number DEAC02-76SF00515.

\section{References}

[1] M. Ibe and R. Kitano, "Sweet Spot Supersymmetry," JHEP 0708, 016 (2007) [arXiv:0705.3686 [hep-ph]].

[2] G. F. Giudice and A. Masiero, "A Natural Solution to the $\mu$ Problem in Supergravity Theories," Phys. Lett. B 206, 480 (1988).

[3] R. Kitano, "Gravitational gauge mediation," Phys. Lett. B 641, 203 (2006) [arXiv:hepph/0607090]. 
[4] M. Dine, W. Fischler and M. Srednicki, "Supersymmetric Technicolor," Nucl. Phys. B 189, 575 (1981); S. Dimopoulos and S. Raby, "Supercolor," Nucl. Phys. B 192, 353 (1981); M. Dine and W. Fischler, "A Phenomenological Model of Particle Physics Based on Supersymmetry,” Phys. Lett. B 110, 227 (1982); "A Supersymmetric Gut," Nucl. Phys. B 204, 346 (1982); C. R. Nappi and B. A. Ovrut, "Supersymmetric Extension of the SU(3) $\times$ $\mathrm{SU}(2) \times \mathrm{U}(1)$ Model," Phys. Lett. B 113, 175 (1982); L. Alvarez-Gaume, M. Claudson and M. B. Wise, "Low-Energy Supersymmetry," Nucl. Phys. B 207, 96 (1982); S. Dimopoulos and S. Raby, "Geometric Hierarchy," Nucl. Phys. B 219, 479 (1983).

[5] M. Dine and A. E. Nelson, "Dynamical supersymmetry breaking at low-energies," Phys. Rev. D 48, 1277 (1993) [arXiv:hep-ph/9303230].

[6] M. Dine, A. E. Nelson and Y. Shirman, "Low-energy dynamical supersymmetry breaking simplified," Phys. Rev. D 51, 1362 (1995) [arXiv:hep-ph/9408384].

[7] M. Dine, A. E. Nelson, Y. Nir and Y. Shirman, "New tools for low-energy dynamical supersymmetry breaking," Phys. Rev. D 53, 2658 (1996) [arXiv:hep-ph/9507378].

[8] M. Ibe and R. Kitano, "Gauge mediation in supergravity and gravitino dark matter," Phys. Rev. D 75, 055003 (2007) [arXiv:hep-ph/0611111].

[9] Y. Nomura and M. Papucci, "A Simple and Realistic Model of Supersymmetry Breaking," arXiv:0709.4060 [hep-ph].

[10] R. Kitano, "Dynamical GUT breaking and $\mu$-term driven supersymmetry breaking," Phys. Rev. D 74, 115002 (2006) [arXiv:hep-ph/0606129].

[11] G. F. Giudice and R. Rattazzi, "Extracting supersymmetry-breaking effects from wavefunction renormalization," Nucl. Phys. B 511, 25 (1998) [arXiv:hep-ph/9706540].

[12] N. Seiberg, "Exact results on the space of vacua of four-dimensional SUSY gauge theories," Phys. Rev. D 49, 6857 (1994) [arXiv:hep-th/9402044].

[13] K. A. Intriligator and P. Pouliot, "Exact superpotentials, quantum vacua and duality in supersymmetric SP(N(c)) gauge theories," Phys. Lett. B 353, 471 (1995) [arXiv:hepth/9505006].

[14] K. A. Intriligator and N. Seiberg, "Duality, monopoles, dyons, confinement and oblique confinement in supersymmetric $\mathrm{SO}(\mathrm{N}(\mathrm{c}))$ gauge theories," Nucl. Phys. B 444, 125 (1995) [arXiv:hep-th/9503179]. 
[15] K. I. Izawa and T. Yanagida, "Dynamical Supersymmetry Breaking in Vector-like Gauge Theories," Prog. Theor. Phys. 95, 829 (1996) [arXiv:hep-th/9602180]; K. A. Intriligator and S. D. Thomas, "Dynamical Supersymmetry Breaking on Quantum Moduli Spaces," Nucl. Phys. B 473, 121 (1996) [arXiv:hep-th/9603158]. 